\title{
A Triangle Division Based Point Matching for Image Registration
}

\author{
Chen Jian ${ }^{\mathrm{a}}$, Zeng Dan , Kang Chen and Zhang Zhijiang \\ Key Laboratory of Specialty Fiber Optics and Optical Access Networks, Shanghai University, \\ Box 145,149 Yanchang Road, Shanghai 200072, China \\ acool_chenjian@163.com
}

Keywords: Image Registration; Feature Matching; Delaunay Based Triangulation; Topology based Matching

\begin{abstract}
One basic requirement of image registration is the high precision of point matching. In this paper, we present a simple and robust method to get more accurate matching points by using the structural information of divided triangles. The method initially construct triangles using Delaunay Triangulation which considering rough matched points as vertices, and then iteratively removes outliers until obtain the consensus topology structure. An algorithm called K-Nearest Neighbor Dis-ratio is also presented to avoiding mismatches. Compared with RANSAC and GTM, Our proposed method acquires more inliers and demonstrates higher matching accuracy.
\end{abstract}

\section{Introduction}

Image registration has been widely applied in remote sensing, medical image analysis, pattern recognition and many other computer vision applications. There are two main matching methods in image registration field: area-based matching and featured-based matching.It is unreliable and also time consuming when getting start from area based matching, so feature based matching has been widely used in image registration job.

S. Umeyama[1] discussed an approximate solution to the Weighted Graph Matching Problem (WGMP) for both undirected and directed graphs. W. Aguilar[2] proposed their point matching method named Graph Transformation Matching (GTM), Their method relies on finding a consensus nearest neighbor graph emerging from candidate matches, and eliminates dubious matches until obtain the final consensus graph. Inspired by GTM, Z. Liu[3] calculated the similarity of two points by comparing local neighbor spatial order using cyclic string matching method.

In this paper, we present a triangle division based point matching method which combining existing feature descriptors with global spatial constraints to remove mismatches and confirm ambiguities. The method firstly constructs triangles using Delaunay triangulation which considering rough matched points as vertices, and then iteratively removes outliers until obtain the consensus topology structure, it can also confirms the ambiguous matches from uncertain matching candidates by comparing their positions with divided triangles relatively.

\section{The Triangle Division Based Matching}

Crude Matching. By improving the NNDR[4] algorithm, we introduce a new method called K-Nearest Neighbor Dis-ratio. It firstly find more than one matching points for every significant point. Suppose $P_{i}$ is a key point in image $1, Q_{j}(j=1,2, \ldots, \mathrm{K})$ is the $j$ th nearest points to $P_{i}$ in image 2, $d_{k}$ is the Euclidean distance for SIFT[5] descriptor between $P_{i}$ and its $k$ th nearest neighbor $Q_{k}$. If $d_{1} / d_{2}>R, d_{1} / d_{3}>R, \ldots, d_{1} / d_{k}>R$ and $d_{1} / d(k+1)<R$, we consider $d_{1}, d_{2}, \ldots, d_{k}$ as the matching candidates of $P_{i}\left(k<K, R\right.$ is a set threshold which determined by processed images), but if $d_{1} / d_{k}$ is still greater than $R$, we give up all these matching candidates and ignore this current point $P_{i}$.

Delaunay Based Triangle Division Matching. Assuming that candidate points in two images have been extracted and their crude mapping couples have been established: $P_{1: 1}$ (one to matching) and $P_{m: n}$ (m to $n$ matching), next step is to find the correct one to one correspondences. Most of our 
images are based on perspective projection model, according camera model, we know that perspective projection of the same plane possess stable structure invariance property. Based on this precondition, our method is mainly to find the matching Delaunay Triangulation geometric topology relationship.

We first remove the false positive and reserve the true positives from the crude one to one matching sets. The theory of judging the true positive or false positive is checking whether the set of matching points is geometrically consistent. The main workflow of removing false positive and reserve true positives can be described with pseudo code as follows:

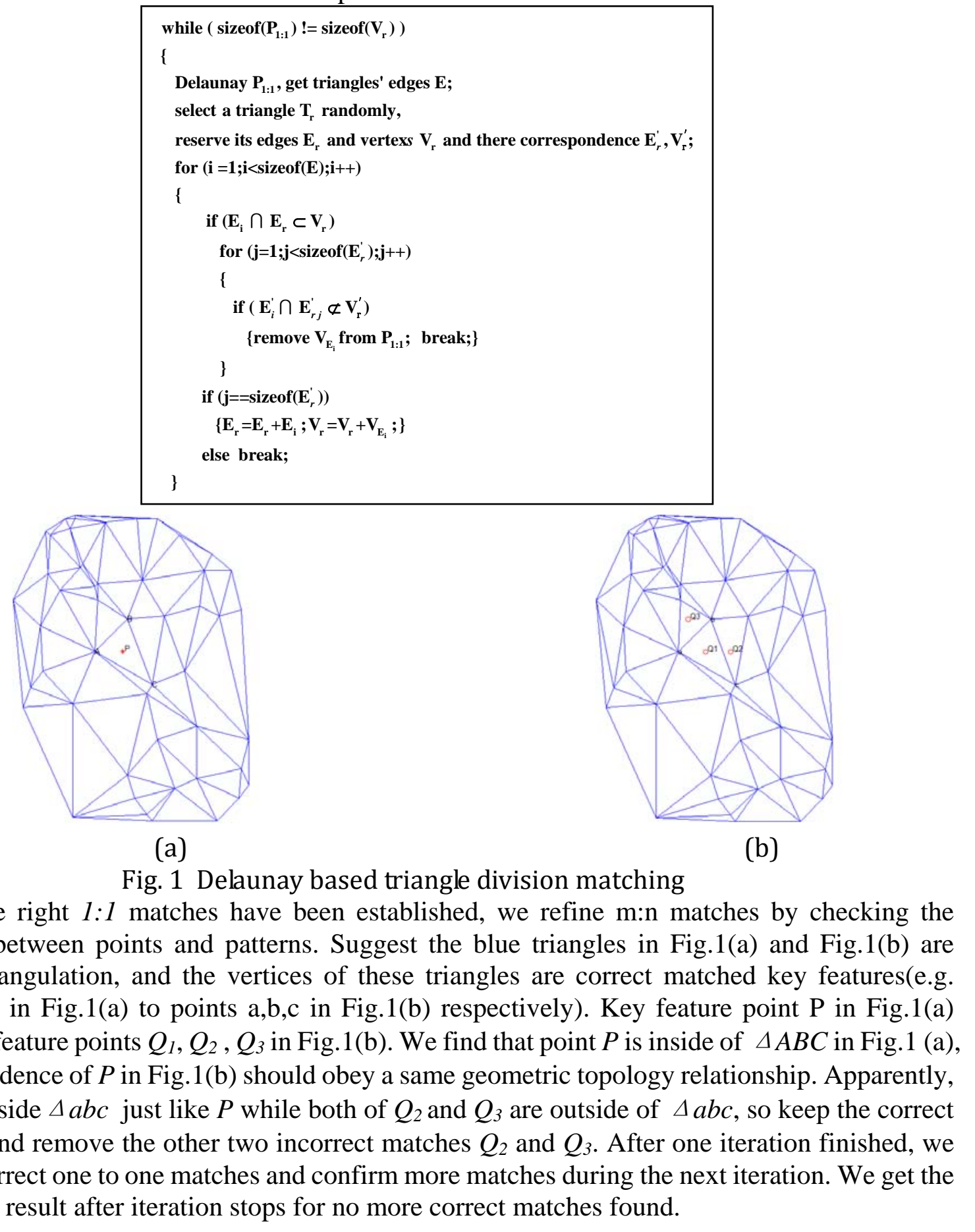

\section{Experiment and Analysis}

In this section, we compare our method against RANSAC and GTM. In RANSAC case, the distance threshold is set to be 5 pixels, the desired probability $p$ of choosing at least one sample free from outliers is set to be 0.99. In the case of GTM, a K-NN graph is created with the value of $\mathrm{K}=3$. 
Planar Matching with Ambiguities. Our first example is to process images with many analogous contents. We selected many image couples contain similar regions. Representatively, teaching buildings matching is a good choice for their windows are very alike mutually.

Fig.2(a) and Fig.2(b) are the GTM figure, we can see that although points in the marked blue circles have same number of adjacent points, they actually are not matched points. Fig.3(a) and Fig.3(b) present the global triangle mapping derived from our method which show a consistent topology distribution.

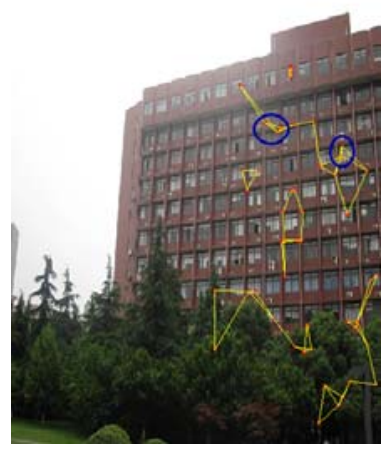

(a)

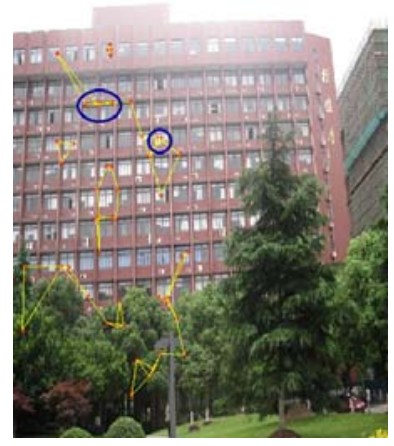

(b)

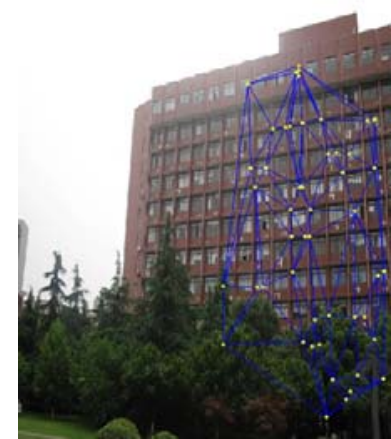

(a)

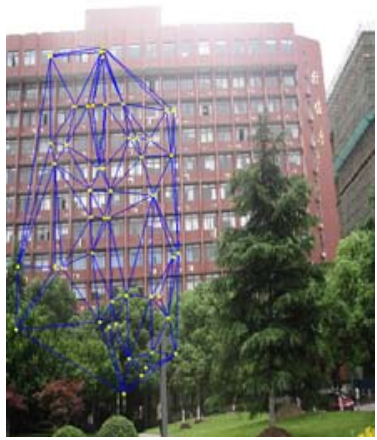

(b)

Fig. 2 GTM matching

Fig. 3 Our Triangulation result

Fig. 4 expresses the recall of these three methods in which $\mathrm{X}$ axis shows the outlier percentage increased from $0 \%$ to $50 \%$. From this graph, we can see that Delauany overwhelms the other two methods obviously. Even the outliers rise to 45\%, Delauany can still obtain more than $80 \%$ inliers. As to GTM algorithm, when the outliers is less than $20 \%$, it can detect about $70 \%$ or more correct matches, but as the outliers grows, its recall value reduces quickly which means it can only obtain less and less useful points. RANSAC performs worst for it can just get less than $50 \%$ correct matching couples.

We also count their precision graph in Fig.5. It can be seen that both Delaunay and RANSAC give high precision value, and it is clear that Delaunay and RANSAC get nearly no mismatches even the under the situation of 50\% outliers. GTM provides a slightly lower precision rate than RANSAC and Delaunay when the outliers are less than 20\%, but it can't keep a good performance while the outliers increasing.

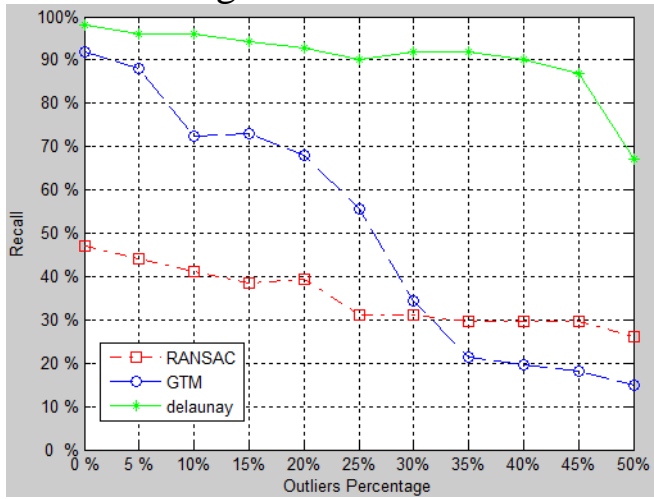

Fig. 4 Recall for planar matching with ambiguities

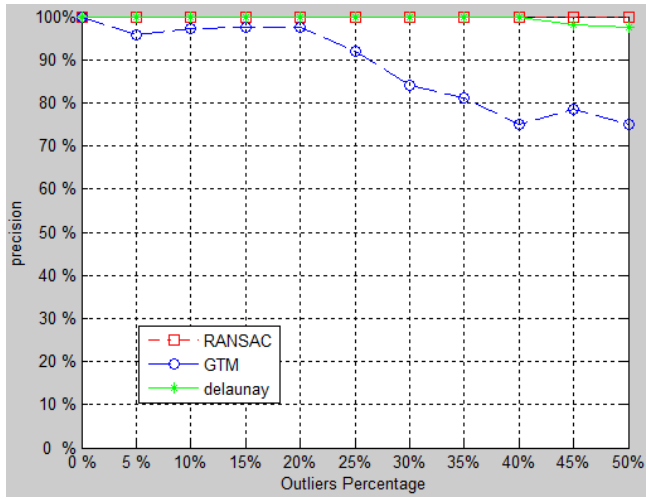

Fig. 5 Precision for planar matching with ambiguities

Multi-planar Matching. When encounter images including multi planes which can't assumed as one plane approximately, RANSAC exposes its shortcoming inevitably for it is base on two-dimensional coordinate transformation rule. In Fig.6(a) and Fig.6(b), the final points obtained by RANSAC gather in the left plane and no points in the right plane are extracted, it means RANSAC can just get seldom points when abundant planes in the scene. Our method can avoid this restriction which can be proved in Fig.7(a) and Fig.7(b), we could see that our method extracts points from both the left and right planes successfully. 


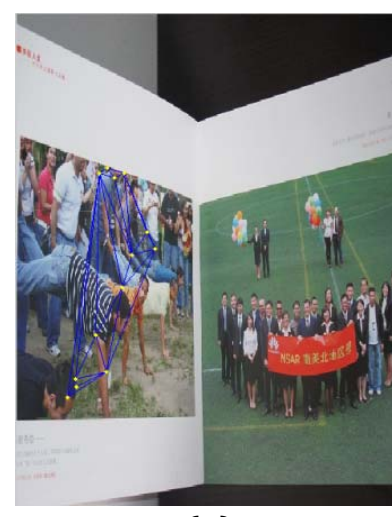

(a)

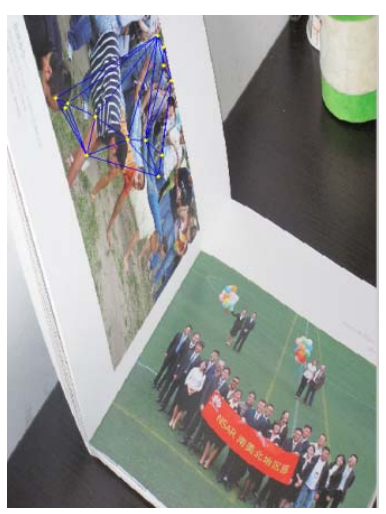

(b)
Fig. 6 RANSAC result of multi-planar matching

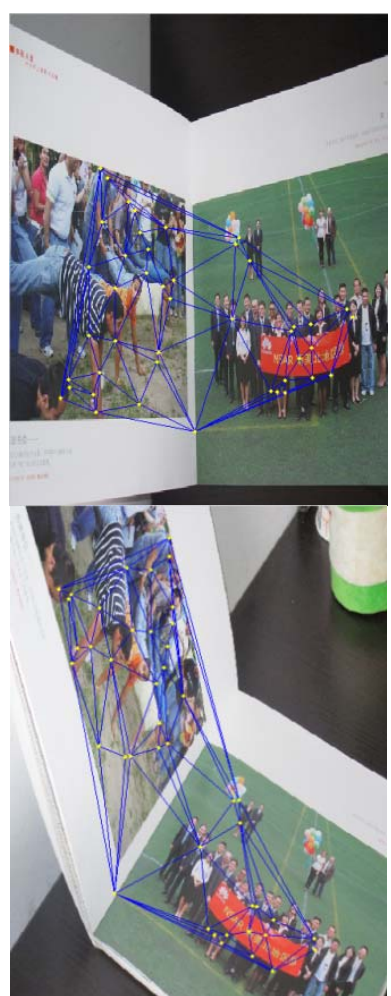

(a)

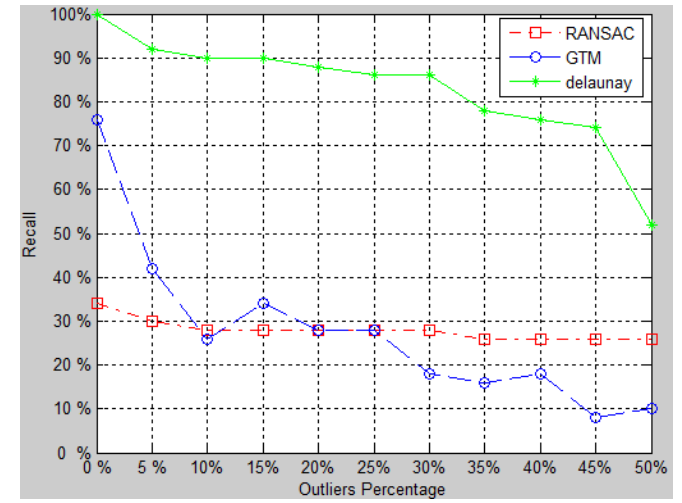

Fig. 8 Recall for multi-planar matching

Fig. 7 Our result of multi-planar matching

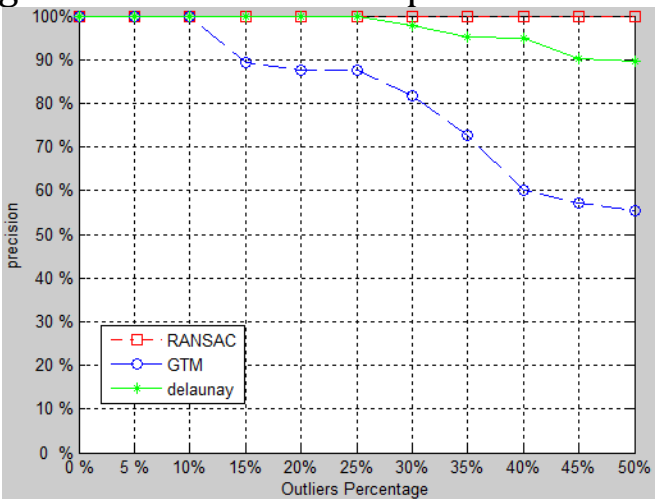

Fig. 9 Precision for multi-planar matching

Fig. 8 and Fig.9 show the recall and precision like previous section. It can be seen that our method provides much higher recall value than RANSAC and GTM, which means our method can get more correct points in multi-planar matching situation. What inspires us is that our method always got a high precision value when the outlier percentage increased from $0 \%$ to $50 \%$.

\section{Conclusion}

In this paper, we have introduced a new method to increase the point matching precision in image registration application. This method is accomplished mainly combine the local features and global graphic distribution relationships. In the experiments, our method exhibited outstanding performance both in the amount of matching points and the accuracy of matching result.

Project (Nos. 40905013 and 60832003) supported by the Na-tional Natural Science Foundation of China, the Shanghai Natural Science Foundation (No. 11ZR1413400), the Key Scientific Research Project of the Shanghai Education Committee (No. 12YZ007).

\section{References}

[1] S. Umeyama. An eigendecomposition approach to weighted graph matching problems. IEEE Trans. Pattern Analysis and Machine Intelligence,vol.10(5),pp.695-703, 1988. 
[2] W. Aguilar, Y. Frauel, F. Escolano, M. E. Martinez-Perez, A. Espinosa-Romero, and M. A. Lozano. A robust Graph Transformation Matching for non-rigid registration. Image and Vision Computing, vol. 27(7), pp. 897-910, 2009.

[3] Z. Liu and Z. Liu. A robust point matching algorithm for image registration. In Proc. 3rd Int. Conf. Mach. Vis., pp. 66-70,2010.

[4] K.Mikolajczyk, C.Schmid. A performance evaluation of local descriptors, IEEE Trans. Pattern Anal. Mach.Intell. Vol.27(10),pp.1615-1630, 2005.

[5] D. G. Lowe. Distinctive image features from scale-invariant keypoints. IJCV, vol.60(2), pp.91-110, 2004. 